\title{
Thermal Pattern and Thermal Tracking: fingerprints of an environmental illicit
}

\author{
by M. Lega*, C. Ferrara*, J. Kosmatka**, G. Persechino*** and R.M.A. Napoli*
}

\author{
* Dept. of Environmental Sciences, University of Naples "Parthenope", Centro Direzionale di Napoli - Isola C4 - \\ 80143, Napoli, Italy, lega@uniparthenope.it \\ ${ }^{* *}$ Dept. of Mechanical and Aerospace Engineering, University of California, San Diego, USA \\ ${ }^{* * *}$ CIRA - Italian Aerospace Research Center
}

\begin{abstract}
Being able to identify crime/guilty relationship is central to police investigation and new technologies enable authorities to do this faster and more accurately than ever before.

In recent years, our research team has introduced the use of a range of aerial platforms and an innovative application of thermography to detect several illegal activities; for example illegal sanitary sewer and storm-drain connections, illicit discharges and other "anomalies" on the surface waters can be easily identified using their thermal infrared signatures.

This paper introduces first results of a Thermal Pattern and Thermal Tracking approach, that can be used to identify different phenomena and several pollutants.
\end{abstract}

\section{Introduction}

Fingerprint evidence plays a crucial role in criminal investigations. Since the person's fingerprints are unique and do not change during the course of their life, they permit to univocally identify a person and place him on the crime scene.

This paper introduces the fingerprint paradigm in the environmental police investigation, defining several specific signatures (pattern) that permit to identify an illicit/anomaly and to find the correlation (tracking) between the crime and the culprit or the source and the target. As on a crime scene it is possible to find different fingerprints of individuals that did not participate to the criminal action, thus it is needed to distinguish the useful ones from the others, in a real environmental scenario, using the proposed methods, our goal is to filter the elements that are not useful and to isolate the phenomenon and the anomalies that are investigated.

Having a good knowledge of the physical phenomena and their effects on the environmental matrixes, it's possible to identify the natural events and the human related in a complex scenario. Moreover, starting from the evidence of an environmental damage, it's possible to search the causes and to define the source-path-target model. In recent years, wide knowledge has been gained by the scientific community about the evidences connected to the analysis of photos and chemical, physical, biological data acquired on a crime scene, but few references on radiometric data (by IR cameras) and their representation exists and particularly on the use of these data and their interpretation for the study and the analysis in environmental police investigations.

If today a conventional investigation begins with the evidence of the crime that defines the scenario to be investigated, in the environmental field the investigation often begins with the evidence of the damage and not of the illegal polluting act. Often the consequences of pollution appear indeed in a different place or a long time after the polluting act has been committed and the correlation between the source and the damage depends from the morphology of the scenario and the physical phenomena that permit the transport of the pollutants. For these reasons the environmental police investigations must be realized alternating a bottom-up and top-down approaches. In order to increase the effectiveness of the investigations, the human senses can be "augmented" using additional advanced sensors and the power of information technology.

In this paper we demonstrate the added value of the use of an integrated approach that combines aerial platforms (e.g. drones), advanced sensors (e.g. IR cameras) and specific IT tools (e.g. multi-view 3D reconstruction and edges detection) in the environmental police investigations. Focal points of this research is the definition of Thermal Pattern and Thermal Tracking as methods to link specific phenomena/anomalies to a IR dataset. 


\section{Materials and Methods}

All data examined in this paper have been acquired by Prof. Massimiliano Lega and his Environmental Engineering Research Team during the activity of Technical Consultant for the Italian Prosecutor Office with the support of several Italian Government CID (Criminal Investigation Departments).

In the recent years the Environmental Engineering Research Team of University Parthenope has introduced new procedures and technologies in the monitoring of contaminated site that are not usual for the domain of the environmental engineering and, particularly, for the forensics field. In the next figures (fig. 1-2) some "shots" of the main platforms scheme and sensors/payload used in our missions already presented with all details in our recent publications [1] are synthetically reintroduced.
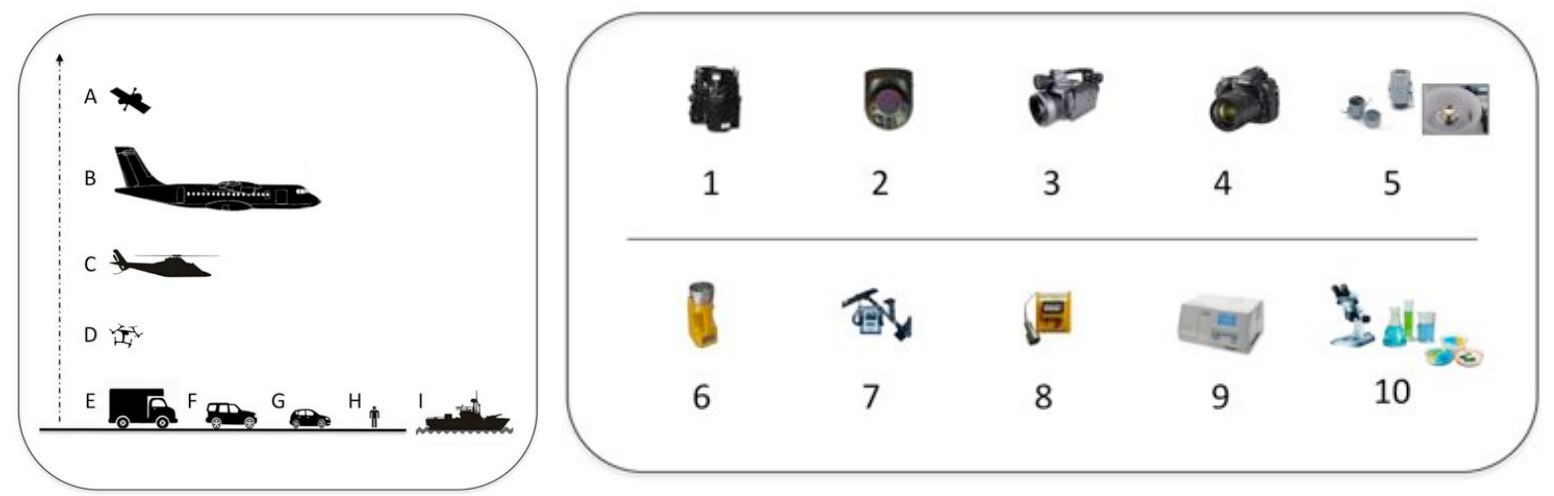

Fig. 1. - (on the left side) Platforms scheme, segmented by quote and environmental matrix (air, land, water): A) Satellite; B) Airplane; C) Rotorcraft; D) Drone; E) Mobile Lab; F) Off-road vehicle; G) Car; H) "human" operator; I) Boat.

- (on the right side) Main Sensors/Payload devices that, potentially, we could use in our missions:

1)Multispectral/Hyperspectral; 2)CCD TV + IR; 3)IR radiometric; 4)DSLR Camera; 5)Air-pollution real-time sensors; 6)Air Sampler; 7)Magnetometer; 8)Geiger counter; 9)Spectrophotometer/spectrometer; 10)Chemical/Physical/Micro-biological tools.
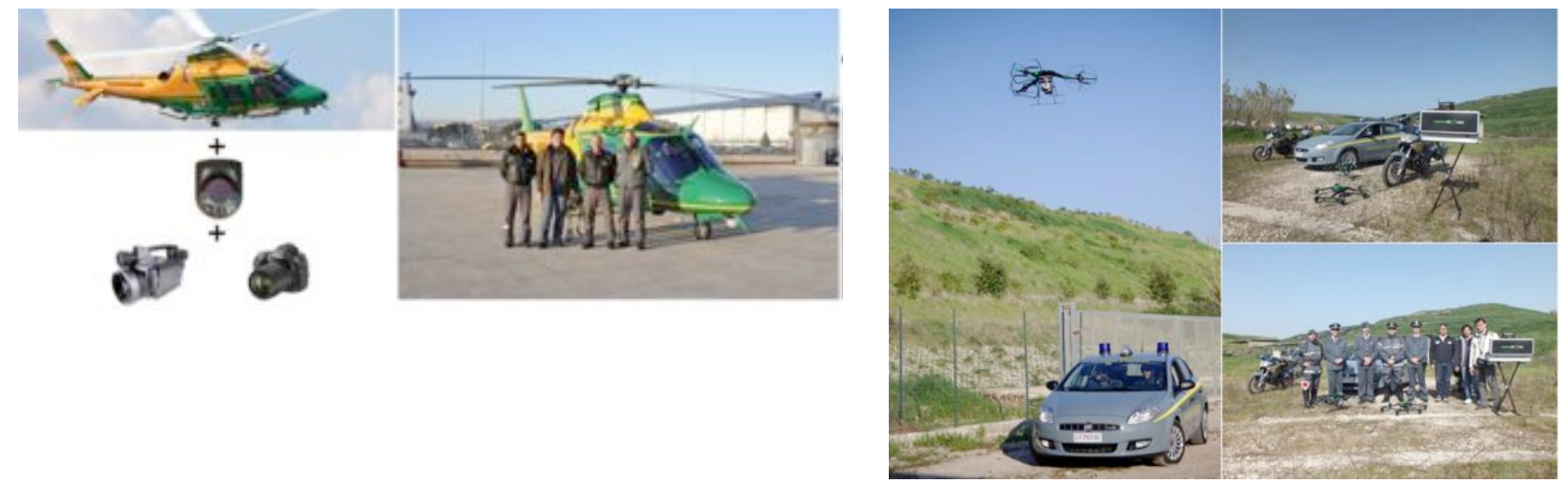

Fig. 2. Example of platform/payload configuration with Italian Financial Police, used in the on the landfills that will be introduced in the next paragraphs:

- (on the left side) Rotorcraft (A109N, A412) + CCD TV + IR + IR radiometric + DSLR Camera; - (on the right side) Drone (Natural Drone StillFly) + car and motorcycles.

The main novelty of our methods lies in the specific procedure that involves aerial infrared thermography, specific information technology tools and combines the data collected at different altitudes with those grabbed by standard methods. Moreover, in the examination of investigated scenario our procedures involve the use of the aerial platforms and in some cases also "special" aerial platforms like a specialized Drone/UAV (Natural Drones "STILLFLY").

The first step of our investigations is data acquisition, based on the aforementioned methods.

The second step is the data processing, analysis and reporting.

Various tools are used to perform the second step and, often, the starting point is the analysis with an advanced image-based 3D modeling software (fig 3 ); in detail, the standard procedure includes:

1) aerial shots alignment, finding the position of the camera for each aerial shot. As a result a "path" point cloud and a set of camera POV (points of view) are created;

2) building model geometry, based on the estimated camera positions and pictures themselves. A 3D polygon mesh, representing the object surface, is built;

3) texturing and/or orthophoto generation. 


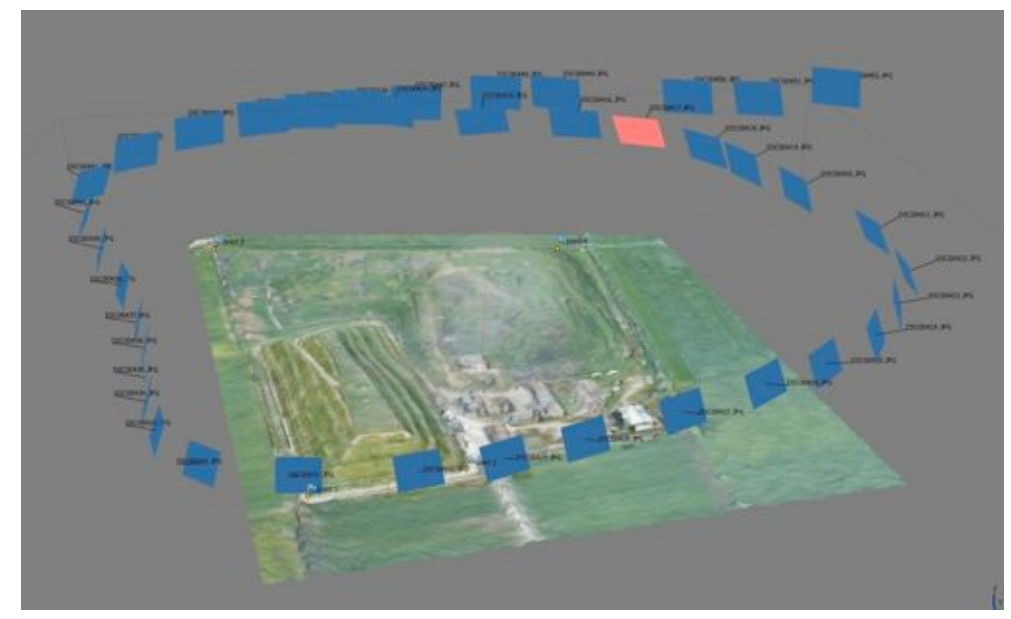

Fig. 3. Multi-view landfills site $3 D$ reconstruction, based on data grabbed by aerial platforms (eg. rotorcraft, drone "Stillfly") and "path" point cloud generation.

$\square$

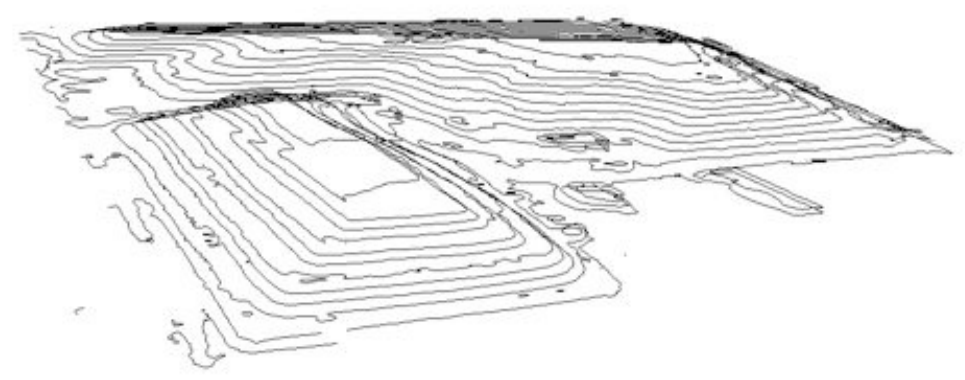

Fig. 4. Example of a landfill site $3 D$ model, represented by contour lines.

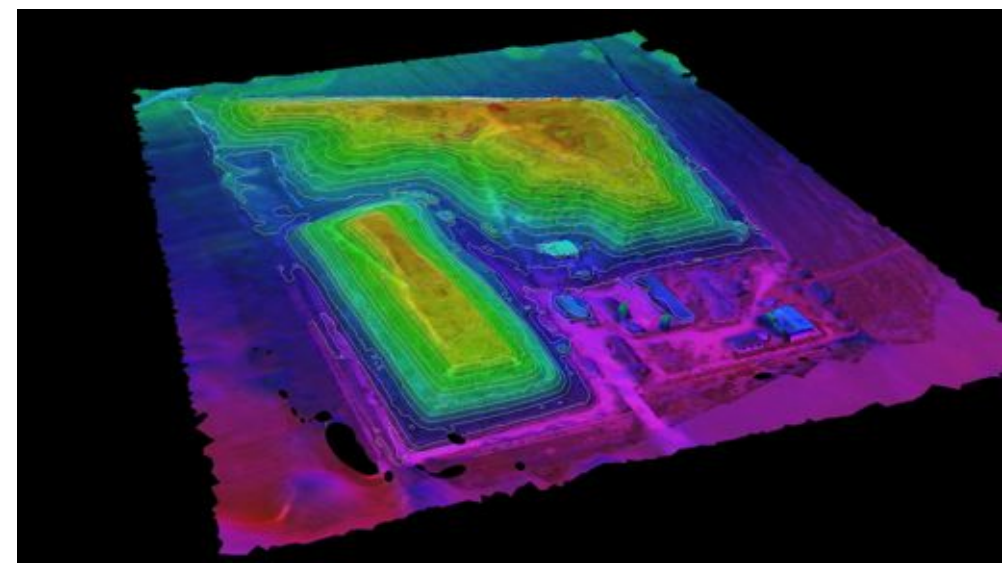

Fig. 5. Example of a landfill site 3D model, represented by a three dimensional contour graph.

Thanks to the reconstruction of the investigated site by a 3D model, it's possible to create a multi-layer structure (fig. 6) that permits to represent in one shot and in overlap all the data acquired during the mission. This approach simplifies the interpretation of data and allows to filter the anomalies related to the specific morphology of the land. 


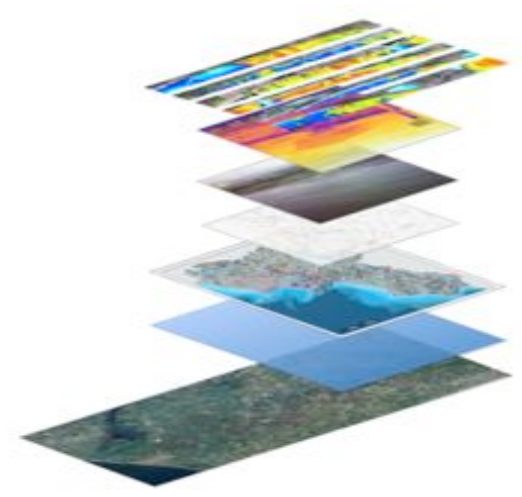

Fig. 6. Example of multi-layer structure.

\subsection{Thermal Pattern}

The human beings perceive information of the surrounding environment by the senses. Using a series of general concepts or patterns that we have learned about the objects as well as with multi-sensorial information and the cognitive ability of recognition we can, for instance, recognize each character of the alphabet, distinguish between male and female faces or identify a known person when hearing a voice on the phone. The aforementioned examples and, in general, all the processes of recognition, involve a classification or an identification of objects, persons, events or situations. Afterwards, a decision is taken and/or a particular action is executed; e.g. the rejection of pieces identified as faulty or damaged in a production line or the provision of an entrance authorization in a security system. The complex and repetitive classification tasks, the necessity of increasing the reliability and objectivity of decisions, judgments or diagnoses and the research works about the human brain promoted the development of algorithms -also called machines - capable of extracting essential knowledge from the environment and representing it mathematically, in order to learn from such a representation a concept of class or category and to identify objects or classify them automatically [2].

Pattern Recognition is an example of advanced object recognition which is influenced by several factors such as shape, reflectance, pose, occlusion and illumination which makes it a difficult task. The choice of the pattern representation is crucial for an effective performance of cognitive tasks such as object recognition and fixation. Pattern recognition aims to classify data (patterns) based on statistical information extracted from the patterns or a priori knowledge [3].

Analyzing different shots of same typology of phenomena we could discover a composite of traits or features characteristic with same geometrics perceptual structure; if the examined pictures are the rendering of IR data (grabbed with an IR Camera) our new concept is: Thermal Pattern. In order to detect a problem in a complex scenario it is important to define several standard thermal pattern related to known phenomenology [4].
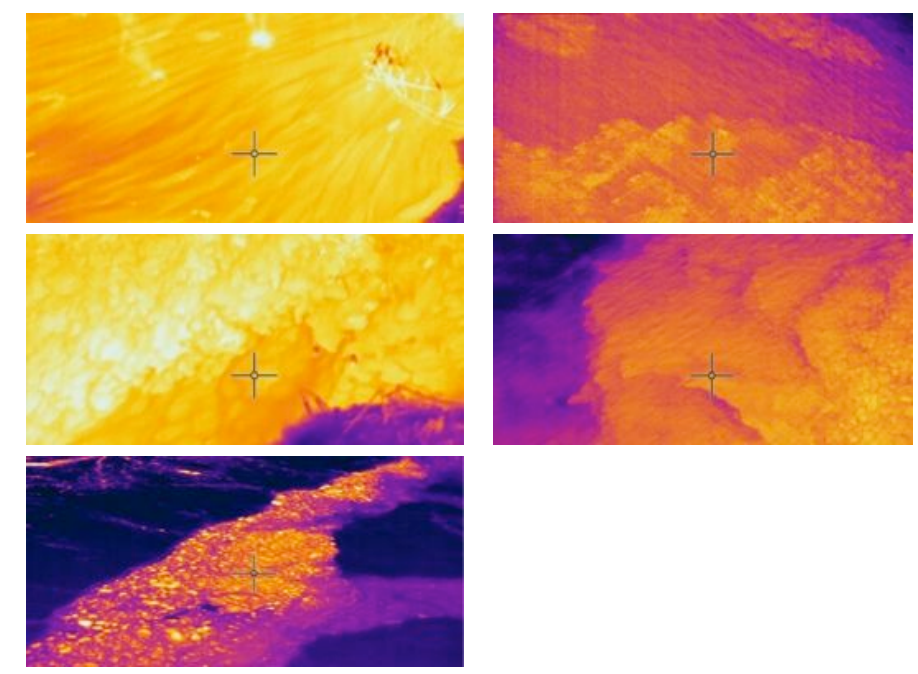

Fig. 7. Thermal Patterns samples (river stream, algal bloom, sea current, etc.) grabbed during our missions. 

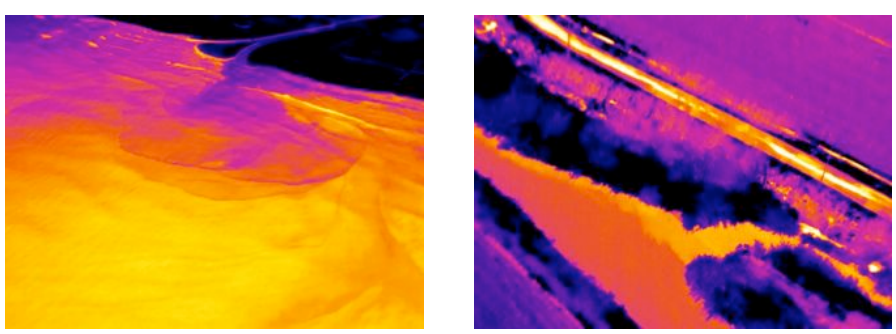

Fig. 8. Example of two different patterns in a complex scenario: plume of the river in the sea (left side) and a tributary in a river (right side).

Not all the examined patterns are only related to the change of temperature but a key role is played by the emissivity/material changes in the same scenario. For example the defined and consistent temperature gap on the shape perimeter permits to suppose also a change in the emissivity value and, consequently, a change of material/pollutants concentration.
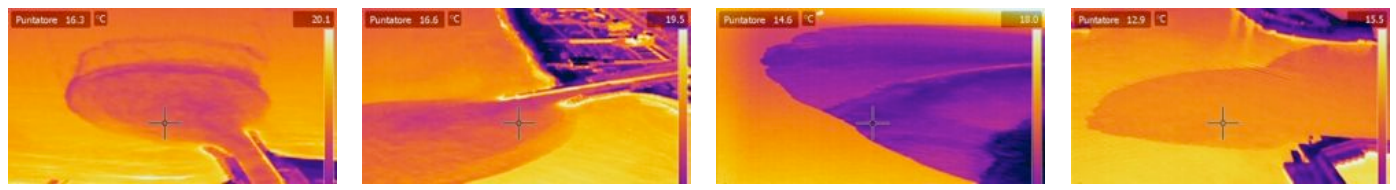

Fig.9. Example of pattern shapes of mixing zones with particular geometry and thermal profile that permits to suppose a change in material/pollutant concentration.

\subsection{Thermal Tracking}

Edge detection refers to the process of identifying and locating sharp discontinuities in an image. The discontinuities are abrupt changes in pixel intensity that characterize boundaries of objects in a scene. Classical methods of edge detection involve convolving the image with an operator (a 2-D filter), which is constructed to be sensitive to large gradients in the image while returning values of zero in uniform regions. There are an extremely large number of edge detection operators available, each designed to be sensitive to certain types of edges. Variables involved in the selection of an edge detection operator include Edge orientation, Noise environment and Edge structure. The geometry of the operator determines a characteristic direction in which it is most sensitive to edges. Operators can be optimized to look for horizontal, vertical, or diagonal edges [5].

Combining edge detection and pattern recognition/tracking ideas, our new concept is: Thermal Tracking.

Using Thermal Tracking pictures/matrixes that are the rendering of IR dataset (grabbed with an IR camera with radiometric output) can be analyzed, links between two or more objects in the same scene can be found and source/path/target correlation can be defined.

As showed in figure 10, using our IR camera carried onboard a rotorcraft, we identified two surface water links between two potential pollutants sources (an industry and a city) and the target (sea).
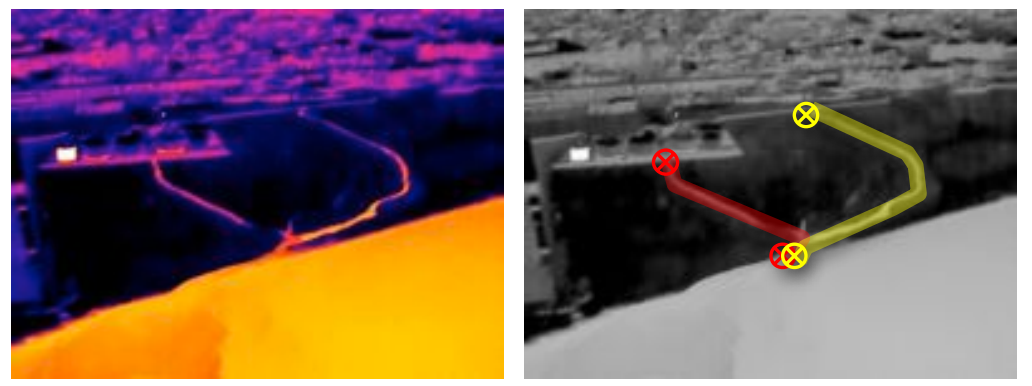

Fig. 10. Thermal Tracking samples: water surfaces links between industry/city and sea (highlighted with red/yellow segments).

Thresholding and edge detection are two image processing techniques which can be used to distinguish image regions and find sudden changes in image intensity. For many real-world scenes and images where there is a clear distinction between foreground and background intensities and objects, these techniques can yield highly favorable results. Herein, two different intelligent thresholding methods and a variety of edge detection methods are tested against a large sequence of imagery in which there exists no real foreground or background, or even true objects. The surface 
structures present in the IR imagery of water surfaces are often very ambiguous with respect to where they begin and end. That is, it is difficult to determine where, exactly, a surface structure clearly exists. The surface structures seen in IR imagery give a visual indication of the state of heat transfer from the water body (i.e., high heat flux vs. low heat flux). The detection of these surface structures can be relevant to remote sensing applications [6].

The Canny, Prewitt, and Sobel operators are all basic and well-studied convolution algoritms/filters which approximate the first spatial derivative and can be used to find edges in an image (fig. 11); thus, we decided to test these operators on our dataset.
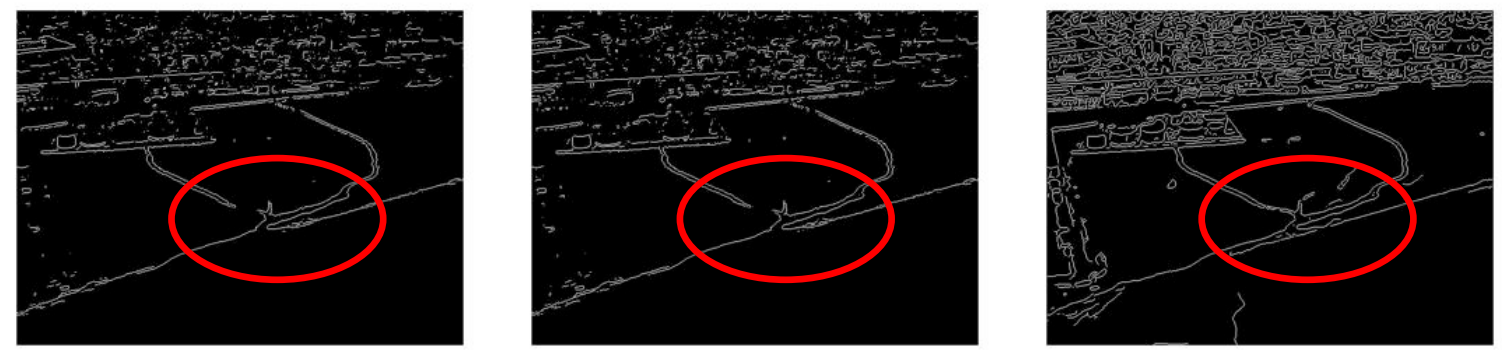

Fig. 11. Comparison of edge detection algorithms/filters: a) Prewitt; b) Sobel; c) Canny.

Qualitatively, the Canny algorithm appears to detect the largest amount of surface structures while the Sobel, Prewitt detectors seemed to be very sensitive to noise in the image. Moreover, the Canny method produces the largest ratio of edge pixels-to-total pixels and finds more edges than the others algorithms.

\section{Results and discussion}

The effectiveness of the use of the proposed integrated system is showed by the success of the investigations.

The proposed procedures, techniques and technologies were indeed tested and validated in environmental police actions directed by Italian Government Bodies (like Public Prosecutor, Environmental Protection Agency, Coast Guard, Financial Police, Italian State Forestry Corps etc.) in real on the field operations and not only in demonstration activities. In detail the environmental criticalities/crimes showed in the next examples were discovered in Campania region (Italy), during several missions in collaboration with Italian Financial Police.

The first example (fig.12) shows the discovery of a link (illegal discharge) between an industry and a river: we flew over the river and noticed a spot anomaly. By the post-processing of the acquired IR data set (Thermal tracking approach), we discovered the link/pollutant path.
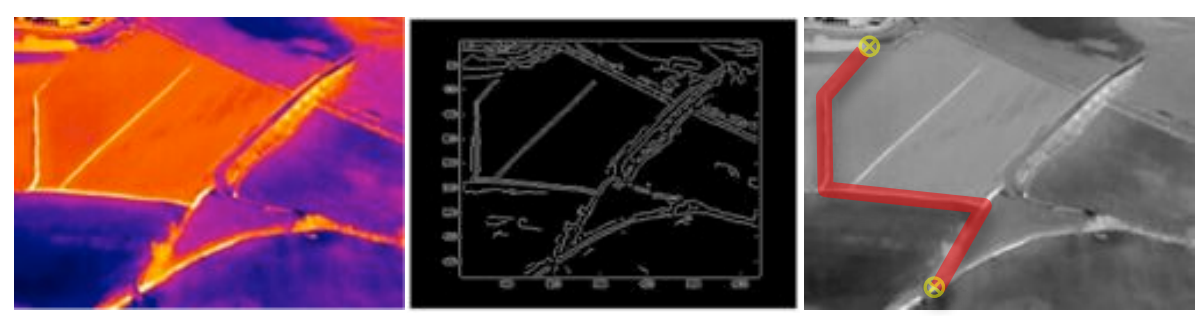

Fig. 12. Example of Thermal Tracking: illegal industry discharge, discovered by the link between industry/river.

The second example (fig.13 to16) refers to an investigation on a site with two adjacent landfills: one legal and the second one illegal. In this case we show the key role of the combination of IR radiometric output and 3D textured polygonal model: thanks to this approach it's possible to relate thermal data with the morphology of the land, thus avoiding mistakes coming from a wrong interpretation of the thermal gradient/gap.

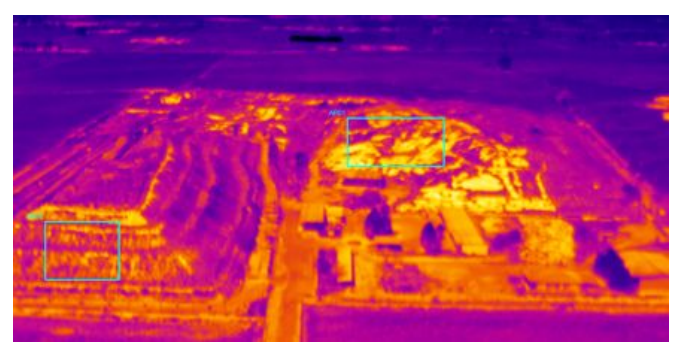

Fig. 13. Example of Thermal pattern recognition: a site with two landfills (one legal and the second one illegal). 


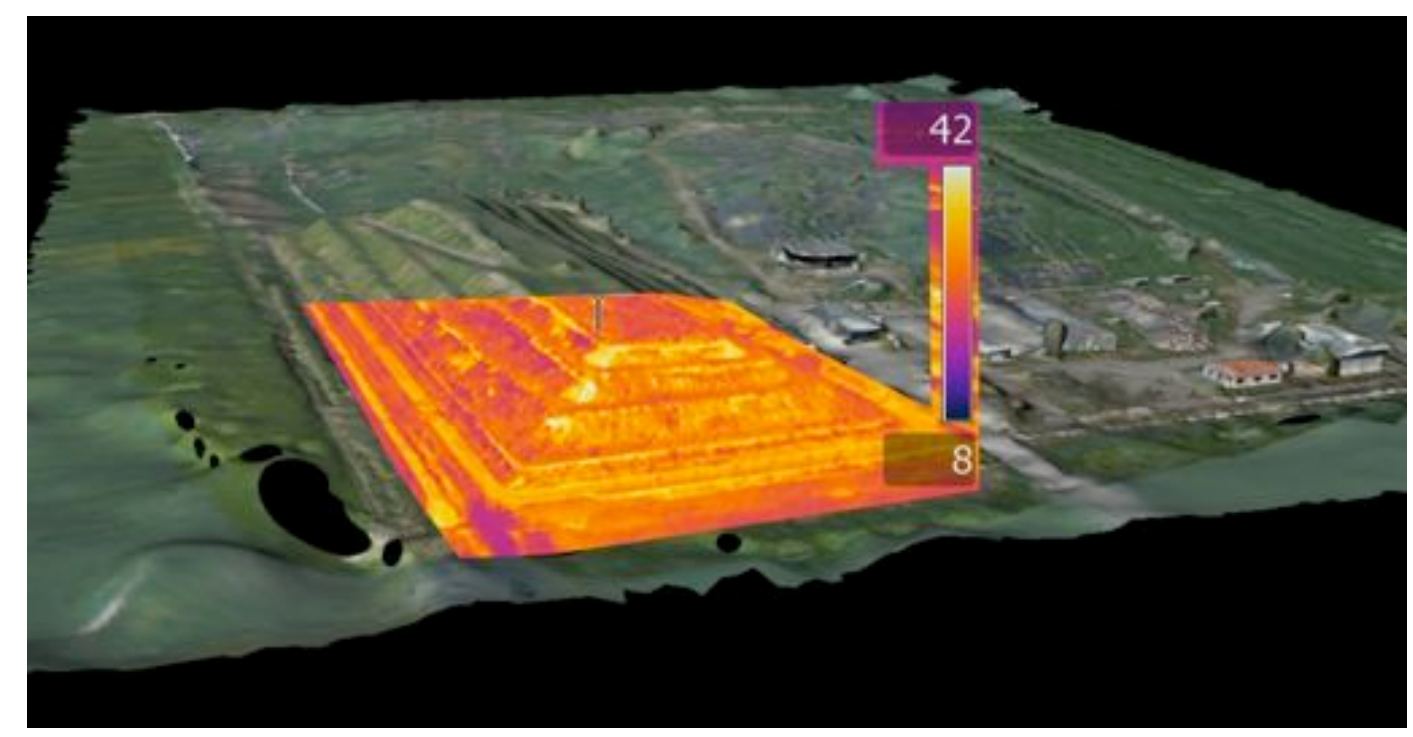

Fig. 14. Combined IR radiometric output/3D textured polygonal model of a landfill.

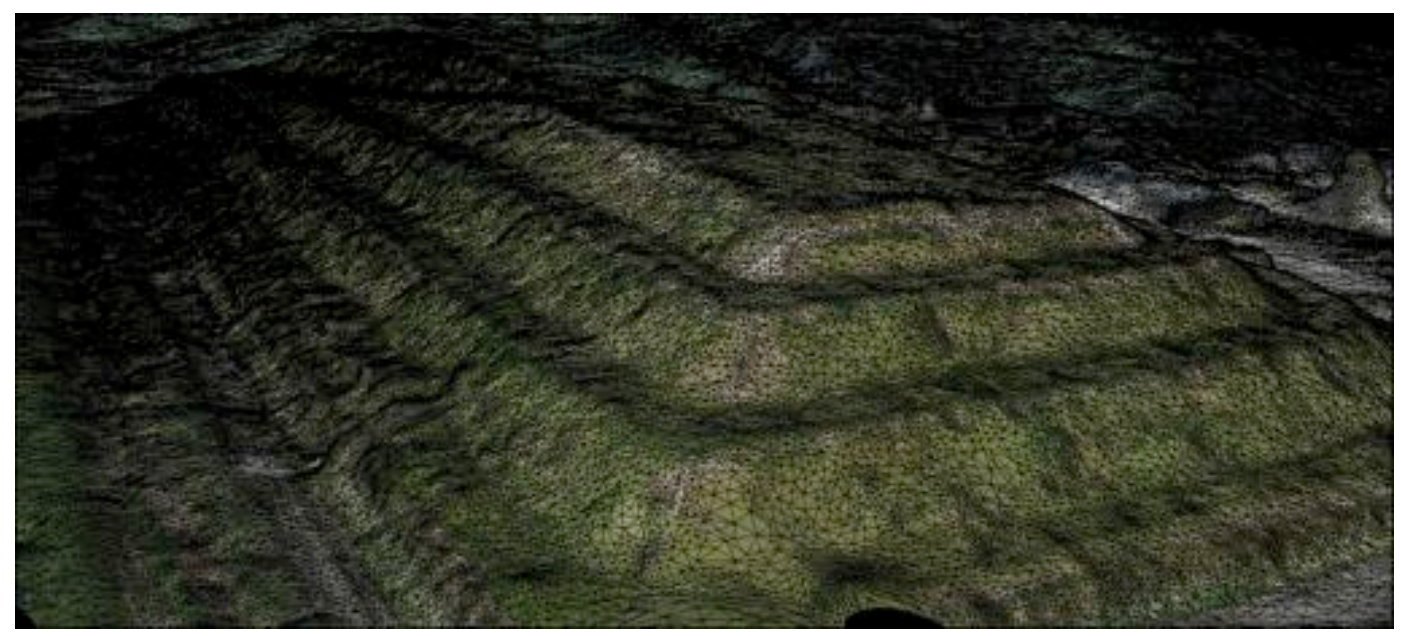

Fig. 15. A detail of 3D textured polygonal model of the examined landfill.

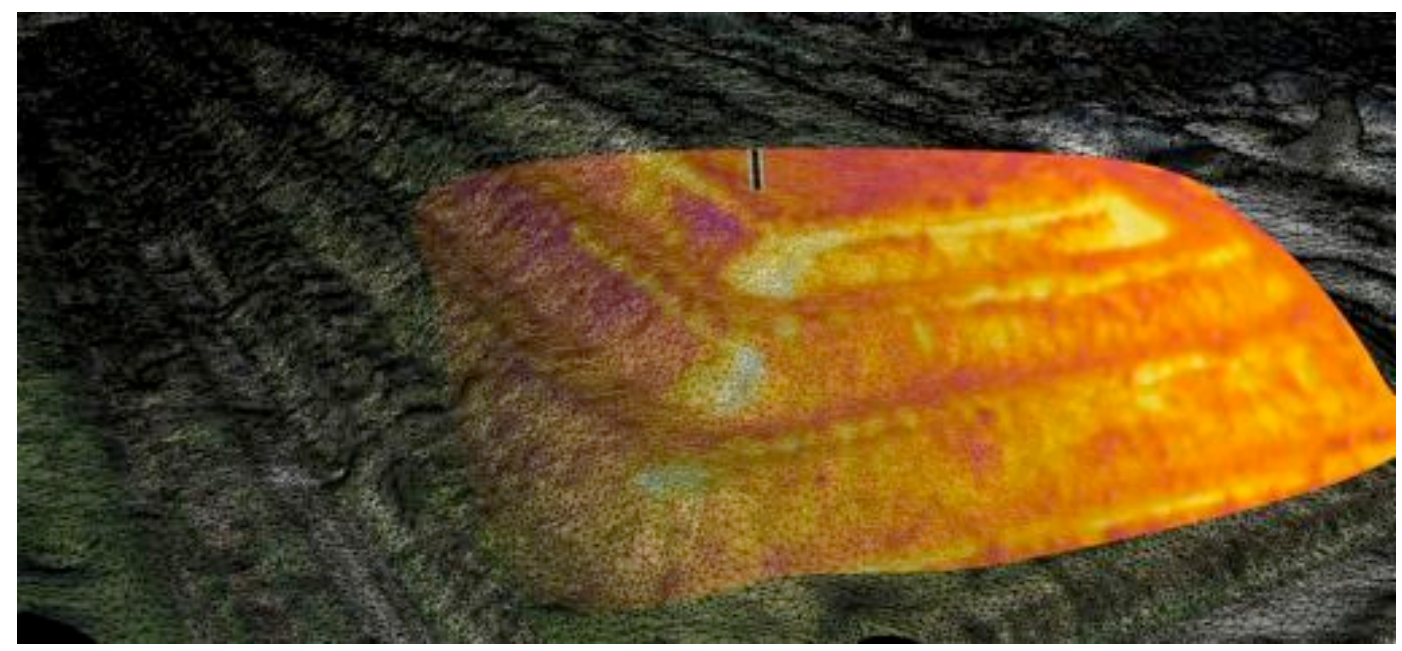

Fig. 16. A detail of the combined IR radiometric output/3D textured polygonal model of the landfill. 


\section{Conclusions}

Being able to identify crime/guilty relationship is central to police investigation and new technologies enable authorities to do this faster and more accurately than ever before.

In recent years, our research team has introduced the use of a range of aerial platforms and an innovative application of thermography to detect several illegal activities; for example illegal sanitary sewer and storm-drain connections, illicit discharges and other "anomalies" on the surface waters can be easily identified using their thermal infrared signatures.

This paper introduces first results of a Thermal Pattern and Thermal Tracking approach, that can be used to identify different phenomena and several pollutants and, moreover, using an accurate statistical analysis, the collected data can be examined to study the pattern recurrence.

Furthermore, current enforcement methodologies employ random and discrete sampling as the principle method for revealing when environmental dumping has occurred. But with this laborious down-stream approach it is difficult to identify an up-stream polluter.

Our studies introduce new methods and technology applications for detecting, evaluating, and tracking signs of environmental contamination using a variety of advanced aerial platforms, a suite of advanced sensors, and new detection software.

In detail, first results of our current studies show that the effectiveness of aerial infrared thermography as tool to detect environmental contamination, can be improved with:

1) the creation of a specific environmental Thermal Patterns database: a data-set of IR images (better radiometric data) connected with typical environmental phenomena. The output of this process permits to identify environmental anomalies and, subsequently, to define a probable environmental pollution;

2) the design of specific software/procedures to find and to augment the path/link between two userdefined targets using the Thermal Tracking. The output of this process permits to identify crime/guilty relationship and gives Police greater power to identify people involved in environmental crimes.

\section{Acknowledgments}

We wish to acknowledge the Italian Public Prosecutor's Office (a special thanks to the Chief Prosecutor Corrado Lembo) and the support of Italian Financial Police.

Finally, we wish to thank NaturalDrones for their contribution in the development of the drone StillFly.

\section{REFERENCES}

[1] Lega M., Kosmatka J., Ferrara C., Russo F., Napoli R.M.A and Persechino G., "Using Advanced Aerial Platforms and Infrared Thermography to Track Environmental Contamination”, Environmental Forensics, 2012 (In Press)

[2] Duda R. O., Hart P. E., and Stork D. G., "Pattern Classification", 2nd ed. New York: Wiley-Interscience, 2001

[3] Bettadapura V., Sai Sharan D. R., "Pattern Recognition with Localized Gabor Wavelet Grids", Proc. IEEE Int. Conf. Computational Intelligence and Multimedia Applications, vol. 2, pp. 517-521, Sivakasi, India, December 2007

[4] Lega M., Napoli R.M.A., "Aerial infrared thermography in the surface waters contamination monitoring". Desalination and Water Treatment, vol. 23, pp. 1-11, 2010

[5] Canny J., "A Computational Approach to Edge Detection". IEEE Transaction on Pattern Analysis and Machine Intelligence, vol8, n.6. , November 1986

[6] Bower S. M., "Structure Detection in Infrared Imagery of Water Surfaces", Mechanical Engineering, Volume: 2, Issue: 1, Pages: 1-5, 2008 